Research Article

\title{
Mathematical Modelling of Lesser Date Moth Using Sex Pheromone Traps and Natural Enemies
}

\author{
Moustafa El-Shahed $\mathbb{D}^{1,2}$ and Asmaa M. Al-Dubiban $\mathbb{D}^{1,2}$ \\ ${ }^{1}$ Department of Mathematics, Unaizah College of Sciences and Arts, Qassim University, P.O. Box 3771, \\ Unaizah 51911, Saudi Arabia \\ ${ }^{2}$ Department of Mathematics, Buraydah College of Sciences and Arts, Qassim University, P.O. Box 1162, \\ Buraydah 51431, Saudi Arabia \\ Correspondence should be addressed to Moustafa El-Shahed; elshahedm@yahoo.com
}

Received 31 August 2020; Revised 25 December 2020; Accepted 2 January 2021; Published 18 January 2021

Academic Editor: Le Anh Tuan

Copyright ( $\odot 2021$ Moustafa El-Shahed and Asmaa M. Al-Dubiban. This is an open access article distributed under the Creative Commons Attribution License, which permits unrestricted use, distribution, and reproduction in any medium, provided the original work is properly cited.

In this paper, a mathematical model for lesser date moth is proposed and analyzed. The interaction between the date palm tree, lesser date moth, and natural enemy has been investigated. The impact of sex pheromone traps on lesser date moth is demonstrated. Some sufficient conditions are obtained to ensure the local and global stability of equilibrium points. The occurrence of local bifurcation near the equilibrium points is performed using Sotomayor's theorem. Theoretical results are illustrated using numerical simulations.

\section{Introduction}

Lesser date moth is one of the most dangerous insect pests affecting date palm around the world. It was discovered in the Gulf region in the mid-eighties in the United Arab Emirates in 1986 and in Saudi Arabia in 1987. It is known that one-third of the production of date crops is lost as a result of the agricultural pests that infect the date palm, especially the lesser date moth [1-5].

Control of the lesser date moth with insecticides is very difficult because it causes tunnels inside the stem of the palm in all directions and depths without any early signs of infection. Several methods have been adopted, including preventive and curative measures to control lesser date moth in date palm plantations around the world, including chemical and nonchemical control, as well as the use of pheromone traps as an effective tool for monitoring and attracting complete insects of lesser date moth. The sex pheromone trap is one of the most important methods used to combat lesser date moth. This method depends on collecting complete insects, killing them to prevent them, from completing their life cycle, and increasing their numbers
$[5,6]$. One of the most promising strategies for controlling lesser date moth is the use of a mating disruption using the sex pheromone traps [7-9]. Larvae predators are among the most important natural enemies that are used in many areas to combat some agricultural pests. Understanding the role of natural enemies in controlling lesser date moth is crucial to the difficulty in using other methods to control it. The lesser date moth is attacked by its natural enemy such as Goniozus swirskiana $[10,11]$. The main objective of this paper is to propose and analyse a mathematical model of lesser date moth with sex pheromone traps taking into consideration the effect of the natural enemy on lesser date moth. The focus will be on the effect of sex pheromone traps parameter on lesser date moth. The paper is arranged as follows: in Section 2 , the mathematical model is described and the boundedness of the solutions of lesser date moth system is verified. The local and global stability of the lesser date moth system is analyzed in Section 3. The local bifurcation conditions are derived in Section 4, and we find the existence of Hopf bifurcation around the coexistence equilibrium point. Finally, some numerical simulations are presented to verify the obtained theoretical results. 


\section{Mathematical Model}

In this section, we construct a mathematical model describing the dynamic of lesser date moth (LDM) population based on its biological and ecological facts.

(i) Let $P(t)$ represent the population density of date palm tree. We assume that the date palm grows with intrinsic growth rate $r$ and carrying capacity $k$ in the absence of lesser date moth.

(ii) We split the life cycle of LDM into two main stages: the larval stage and the adult stage. The larval stage is the most dangerous, as it feeds on the living tissue inside the trunk of the palm tree, which leads to the death of the date palm tree. Let $L(t)$ represent the population density of the lesser date moth larvae. The adult stage is split into two compartments: the lesser date moth female $F(t)$ and lesser date moth male $M(t)$.

(iii) Let $W(t)$ represent the population density of natural enemy at time $t$.

(iv) The lesser date moth larvae emerge to female or male at the rate of $\alpha$. Among lesser date moth larvae which emerge, we suppose that a proportion $\epsilon$ becomes female, while complimentary part $(1-\varepsilon)$ becomes male.

(v) We consider the lesser date moth response on date palm as Holling type-II response function $\left(\left(\beta_{1} P\right) /(a+P)\right)$, which denotes the rate of consumption of date palm per LDM. $\beta_{1}$ is the predation rate of a date palm tree by lesser date moth. The parameter $a$ is the half-saturation point, i.e., the value of $P$ at which the predation rate is half the maximum value. $e_{1}$ is the conversion rate of date palm biomass to LDM biomass, and we assume $0<e_{1}<1$ since the whole biomass of the date palm is not transformed to the biomass of the lesser date moth. It is also assumed that their natural enemy attacks the lesser date moth larvae, i.e., Goniozus swirskiana population, at a rate of $\beta_{2}$, following Holling type-II functional response and $b$ is the value of $L$ at which the predation rate is half the maximum value. $e_{2}$ is the conversion rate of lesser date moth biomass to natural enemy biomass and $0<e_{2}<1$.

(vi) In order to maintain a low level of lesser date moth, we consider controlling the use of female pheromone traps to disrupt male mating behavior. Firstly, mating between LDM males and LDM females is disturbed to reduce the chances of fertilization, which in turn reduces the number of offsprings. This is done using traps that are releasing a female pheromone lure to which males are attracted. This leads to a decrease in the number of males available for mating near females and reduces the chance of fertilization. Secondly, the LDM males, attracted with traps, are killed because traps contain pesticides that kill what is caught.

(vii) To take into account the effect of pheromone traps, we consider the approach proposed by Barclay and Van den Driessche [12], Barclay and Hendrichs [13], and Anguelov et al. [14]. That is, the strength of the trap is represented by the amount of pheromones released by an equivalent number of wild females. Following [14-17], we assume that the effect of the pheromone trap corresponds to the attraction of additional $\eta$ females. In such a context, the total number of LDM females attracting LDM males is $F+\eta$. In particular, this means that males have a probability of $(\eta /(F+\eta))$ to be attracted to pheromone traps. The parameter $\gamma$ represents the mortality rate of males attracted to pheromones traps.

The model of lesser date moth with sex pheromone trap is described by the following system:

$$
\begin{aligned}
\frac{\mathrm{d} P}{\mathrm{~d} t} & =r P\left(1-\frac{P}{k}\right)-\frac{\beta_{1} P L}{a+P}-\mu_{1} P, \\
\frac{\mathrm{d} L}{\mathrm{~d} t} & =\frac{e_{1} \beta_{1} P L}{a+P}-\alpha L-\frac{\beta_{2} L W}{b+L}-\mu_{2} L, \\
\frac{\mathrm{d} F}{\mathrm{~d} t} & =\varepsilon \alpha L-\mu_{3} F, \\
\frac{\mathrm{d} M}{\mathrm{~d} t} & =(1-\varepsilon) \alpha L-\frac{\gamma \eta M}{F+\eta}-\mu_{4} M, \\
\frac{\mathrm{d} W}{\mathrm{~d} t} & =\frac{e_{2} \beta_{2} L W}{b+L}-\mu_{5} W,
\end{aligned}
$$

with initial values $P(0)=P_{0} \geq 0, L(0)=L_{0} \geq 0, F$ $(0)=F_{0} \geq 0, M(0)=M_{0} \geq 0, W(0)=W_{0} \geq 0$. The variables and parameters of system (1) are presented in Table 1.

2.1. Nonnegativity and Boundedness. The boundedness of the solutions of model (1) is given in the following.

Proposition 1. The population density of date palm tree $P$ is always bounded from above by $P_{1}=(k / r)\left(r-\mu_{1}\right)$.

Proof. According to the first equation of the lesser date moth system $(1)$, one has $(\mathrm{d} P / \mathrm{d} t) \leq r P(1-(P / k))$, as a result $(\mathrm{d} P / \mathrm{d} t) \leq-(r / k)\left(P^{2}-(k / r)\left(r-\mu_{1}\right) P\right)$, then we have

$$
\left.\frac{\mathrm{d} P}{\mathrm{~d} t}+\left(r-\mu_{1}\right) P \leq-\frac{r}{k}\left(P^{2}-\frac{2 k}{r}\left(r-\mu_{1}\right) P\right)\right) \leq-\frac{k}{r}\left(r-\mu_{1}\right)^{2} .
$$
$P \leq P_{1}$.
Applying the comparison theorem, one obtains 
TABLE 1: Biological description of parameters used in system (1).

\begin{tabular}{lc}
\hline Parameters & Description \\
\hline$r$ & The date palm tree intrinsic growth rate \\
$k$ & The carrying capacity \\
$\alpha$ & Transfer rate from larvae to adult lesser date moth \\
$\beta_{1}$ & Predation intensity between date palm and larvae \\
$\beta_{2}$ & Predation intensity between larvae and natural \\
& enemy \\
$e_{1}$ & Conversion coefficient for predation term between $P$ \\
$e_{2}$ & and $L$ \\
$\mu_{1}$ & Conversion coefficient for predation term between $L$ \\
$\mu_{2}$ & and $W$ \\
$\mu_{3}$ & Mortality rate of date palm tree \\
$\mu_{4}$ & Mortality rate of lesser date moth larvae \\
$\mu_{5}$ & Mortality rate of lesser date moth female \\
$a, b$ & Mortality rate of natural enemy \\
$\eta$ & Half-saturation constants \\
$\gamma$ & Maximal death rate by sex pheromone trap \\
\hline
\end{tabular}

Theorem 1. All the solutions of lesser date moth model (1) starting in $\mathbb{R}_{+}^{5}$ are uniformly bounded.

Proof. Following [18-21], let $(P(t), L(t), F(t), M(t), W(t))$ to be any solution of the system (1) with nonnegative initial conditions. Let $H(t)=P(t)+L(t)+F(t)+M(t)+W(t)$, then

$$
\begin{aligned}
\frac{\mathrm{d} H}{\mathrm{~d} t} & \leq r P\left(1-\frac{P}{k}\right)-\mu_{1} P-\mu_{2} L-\mu_{3} F-\mu_{4} M-\mu_{5} W-\frac{\gamma \eta M}{F+\eta} \\
& \leq r P\left(1-\frac{P}{k}\right)-v H, \\
& \leq \frac{-r}{k}\left(P^{2}-k P\right)-v H, \\
& \leq \frac{-r}{k}\left(P-\frac{k}{2}\right)^{2}+\frac{r k}{4}-\nu H,
\end{aligned}
$$

where $v=\min \left\{\mu_{1}, \mu_{2}, \mu_{3}, \mu_{4}, \mu_{5}\right\}$. Thus, $(\mathrm{d} H / \mathrm{d} t)+v M \leq$ $((r k) / 4)$. Applying the comparison theorem, one obtains $0 \leq H(t) \leq((r k) / 4 v)$. Hence, all the solutions of lesser date moth system (1) that start in $\mathbb{R}_{+}^{5}$ are uniformly bounded in the region

$$
\Omega=\left\{(P, L, F, M, W) \in \mathbb{R}_{+}^{5}: H \leq \frac{r k}{4 \nu}+\xi \text {, for any } \xi>0\right\} .
$$

Using the next generation method [22], one can obtain the basic offspring number

$$
R_{0}=\frac{e_{1} \beta_{1} P_{1}}{\left(\alpha+\mu_{2}\right)\left(a+P_{1}\right)}
$$

\section{Equilibria and Stability}

The lesser date moth model (1) has the following three equilibrium points:

(1) $E_{0}=(0,0,0,0,0)$, which always exists.

(2) The free lesser date moth equilibrium point $E_{1}=\left(P_{1}, 0,0,0,0\right) . E_{1}$ exists if $r>\mu_{1}$.

(3) The free natural enemy equilibrium point $E_{2}=\left(P_{2}, L_{2}, F_{2}, M_{2}, 0\right)$, where

$$
\begin{aligned}
P_{2} & =\frac{a\left(\alpha+\mu_{2}\right)}{e_{1} \beta_{1}-\left(\alpha+\mu_{2}\right)} \\
L_{2} & =\frac{a+P_{2}}{\beta_{1}}\left(r-\mu_{1}-\frac{P_{2}}{k}\right), \\
F_{2} & =\frac{\varepsilon \alpha L_{2}}{\mu_{3}} \\
M_{2} & =\frac{(1-\varepsilon) \alpha L_{2}\left(\eta+F_{2}\right)}{\gamma \eta+\mu_{4}\left(\eta+F_{2}\right)} .
\end{aligned}
$$

The free natural enemy equilibrium point exists positively if $R_{0}>1$.

(4) The coexistence equilibrium point $E_{3}=\left(P_{3}\right.$, $\left.L_{3}, F_{3}, M_{3}, W_{3}\right)$, where

$$
\begin{aligned}
L_{3} & =\frac{b \mu_{5}}{e_{2} \beta_{2}-\mu_{5}}, \\
F_{3} & =\frac{\varepsilon b \alpha \mu_{5}}{\mu_{3}\left(e_{2} \beta_{2}-\mu_{5}\right)}, \\
M_{3} & =\frac{(1-\varepsilon) \alpha\left(F_{3}+\eta\right)}{\eta \gamma+\mu_{4}\left(F_{3}+\eta\right)}, \\
P_{3} & =\frac{-a r-k \mu_{1}+k r+\sqrt{\left(r(a+k)-k \mu_{1}\right)^{2}-4 k r \beta_{1} L_{3}}}{2 r}, \\
W_{3} & =\frac{\left(b+L_{3}\right)\left[e_{1} \beta_{1} P_{3}-\left(\alpha+\mu_{2}\right)\left(a+P_{3}\right)\right]}{\beta_{2}\left(a+P_{3}\right)} .
\end{aligned}
$$

The coexistence equilibrium point $E_{3}$ exists if $e_{2} \beta_{2}>\mu_{5}$ and $e_{1} \beta_{1} P_{3}>\left(\alpha+\mu_{2}\right)\left(a+P_{3}\right)$.

The locally asymptotically stable equilibrium points of lesser date moth system (1) are now investigated. Following $[23,24]$, the Jacobian matrix is given as follows: 


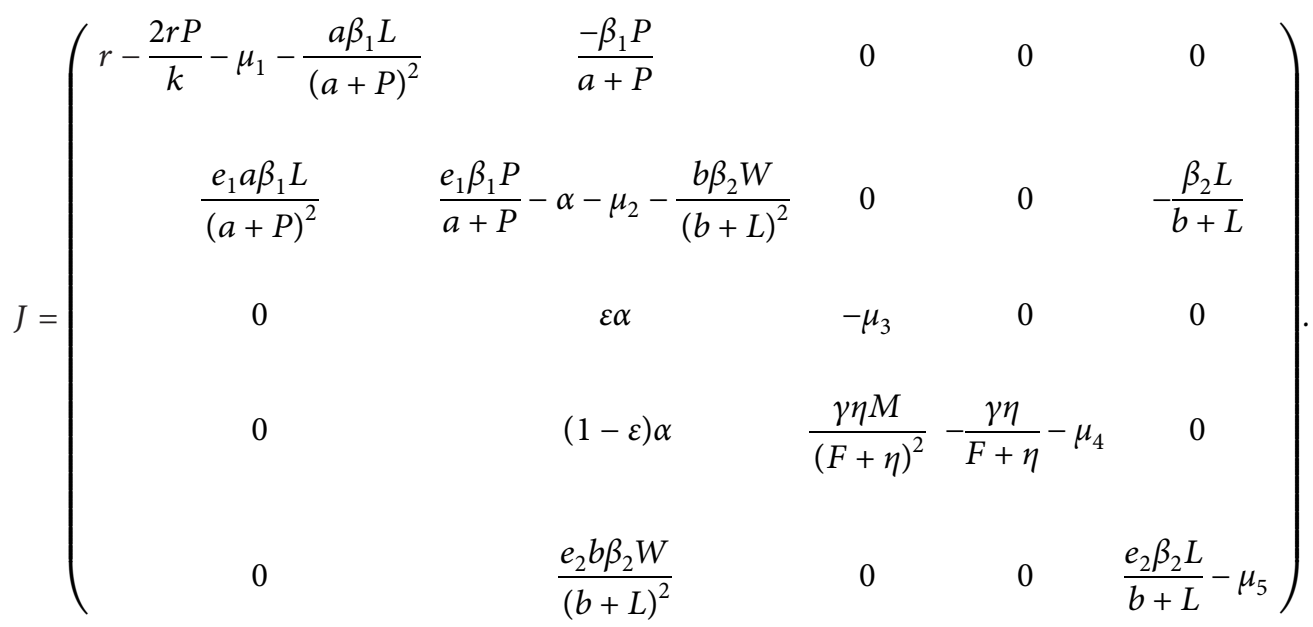

The stability of lesser date moth extinction equilibrium point $E_{0}=(0,0,0,0,0)$ is investigated as follows.

Theorem 2. If $r<\mu_{1}$, then $E_{0}$ is locally asymptotically stable.

Proof. The Jacobian matrix of LDM system (1) at $E_{0}$ is

$$
J\left(E_{0}\right)=\left(\begin{array}{ccccc}
r-\mu_{1} & 0 & 0 & 0 & 0 \\
0 & -\left(\alpha+\mu_{2}\right) & 0 & 0 & 0 \\
0 & \varepsilon \alpha & -\mu_{3} & 0 & 0 \\
0 & (1-\varepsilon) \alpha & 0 & -\left(\gamma+\mu_{4}\right) & 0 \\
0 & 0 & 0 & 0 & -\mu_{5}
\end{array}\right) .
$$

The eigenvalues of $J\left(E_{0}\right)$ are $\lambda_{1}=r-\mu_{1}, \lambda_{2}=-\left(\alpha+\mu_{2}\right)$, $\lambda_{3}=-\mu_{3}, \lambda_{4}=-\left(\gamma+\mu_{4}\right)$, and $\lambda_{5}=\mu_{5}$. When $r<\mu_{1}$, all the eigenvalues of the Jacobian matrix $J\left(E_{0}\right)$ near the equilibrium point $E_{0}$ have negative real parts. Thus, due to the Routh-Hurwitz criterion, $E_{0}$ is locally asymptotically stable if $r<\mu_{1}$.

Theorem 3. If $r<\mu_{1}$, then $E_{0}$ is globally asymptotically stable.

Proof. Considering the following positive definite Lyapunov function $\Psi_{1}(t)=P(t)+L(t)+F(t)+M(t)+W(t)$, then the time derivative of $\Psi_{1}$ along the solution of lesser date moth system (1), one obtains

$$
\begin{aligned}
\frac{d \Psi_{1}}{d t} \leq & r P\left(1-\frac{P}{k}\right)-\mu_{1} P-\mu_{2} L-\mu_{3} F \\
& -\mu_{4} M-\mu_{5} W-\frac{\gamma \eta M}{F+\eta} \leq\left(r-\mu_{1}\right) P .
\end{aligned}
$$

Choosing $r<\mu_{1}$, one obtains $(\mathrm{d} P / \mathrm{d} t) \leq 0$. Thus, if $r<\mu_{1}$, then $E_{0}$ is globally asymptotically stable.

The stability of free lesser date moth equilibrium point $E_{1}=\left(P_{1}, 0,0,0,0\right)$ is investigated as follows.
Theorem 4. If $R_{0}<1$, then free lesser date moth equilibrium point $E_{1}$ is locally asymptotically stable.

Proof. The Jacobian matrix of model (1) at $E_{1}$ is

$$
J\left(E_{1}\right)=\left(\begin{array}{ccccc}
r-\frac{2 r P_{1}}{k}-\mu_{1} & \frac{-\beta_{1} P_{1}}{a+P_{1}} & 0 & 0 & 0 \\
0 & \frac{e_{1} \beta P_{1}}{a+P_{1}}-\left(\alpha+\mu_{2}\right) & 0 & 0 & 0 \\
0 & \varepsilon \alpha & -\mu_{3} & 0 & 0 \\
0 & (1-\varepsilon) \alpha & 0 & -\gamma-\mu_{4} & 0 \\
0 & 0 & 0 & 0 & -\mu_{5}
\end{array}\right) .
$$

The eigenvalues of $J\left(E_{1}\right)$ are $\lambda_{1}=-\mu_{5}, \lambda_{2}=-\mu_{3}$, $\lambda_{3}=-\left(\gamma+\mu_{4}\right)$, and $\lambda_{4}=-\left(r+\mu_{1}\right)$. It can be observed that $\lambda_{5}<0$, when $\left(\left(e_{1} \beta P_{1}\right) /\left(a+P_{1}\right)\right)<\left(\alpha+\mu_{2}\right)$ which is equivalent to $R_{0}<1$. So, $E_{1}$ is locally asymptotically stable if $R_{0}<1$.

Theorem 5. If $e_{1} \beta_{1} P_{1}<a \mu_{2}$, then equilibrium point $E_{1}$ is globally asymptotically stable.

Proof. The following positive definite Lyapunov function is considered:

$$
\begin{aligned}
\Psi_{2}(t)= & e_{1}\left(P-P_{1}-P_{1} \ln \left(\frac{P}{P_{1}}\right)\right)+\frac{1}{2} L^{2}+L(t) \\
& +F(t)+M(t)+\frac{1}{e_{2}} W(t) .
\end{aligned}
$$

By calculating the time derivative of $\Psi_{2}$ along the solution of system (1), one obtains 


$$
\begin{aligned}
\frac{d \Psi_{2}}{d t} & \leq e_{1}\left(P-P_{1}\right)\left[r\left(1-\frac{P}{k}\right)-\mu_{1}-\frac{\beta_{1} L}{a+P}\right]+L^{2}\left(\frac{e_{1} \beta_{1} P}{a+P}-\alpha-\mu_{2}-\frac{\beta_{2} W}{b+L}\right)+\frac{e_{1} \beta_{1} P L}{a+P}-\mu_{2} L \\
& \leq \frac{-e_{1} r}{k}\left(P-P_{1}\right)^{2}+\left(\frac{e_{1} \beta_{1} P_{1}}{a}-\mu_{2}\right) L+L^{2}\left(\frac{e_{1} \beta_{1} P_{1}}{a+P_{1}}-\alpha-\mu_{2}\right)-\frac{\beta_{2} W L^{2}}{b+L} \\
& \leq \frac{-e_{1} r}{k}\left(P-P_{1}\right)^{2}+\left(\frac{e_{1} \beta_{1} P_{1}}{a}-\mu_{2}\right) L+\left(\alpha+\mu_{2}\right)\left(R_{0}-1\right) L^{2} .
\end{aligned}
$$

Choosing $e_{1} \beta_{1} P_{1}<a \mu_{2}$, one obtains $\left(\mathrm{d} \Psi_{2} / \mathrm{d} t\right) \leq 0$, and hence, the free lesser date moth equilibrium point $E_{1}$ is globally asymptotically stable.

The stability of the free natural enemy equilibrium point $E_{2}=\left(P_{2}, L_{2}, F_{2}, M_{2}, 0\right)$ is investigated as follows.
Theorem 6. If $\left(\left(\beta_{1} L_{2}\right) /\left(a+P_{2}\right)^{2}\right)<(r / k)$ and $\left(\left(e_{2} \beta_{2} L_{2}\right) /\right.$ $\left.\left(b+L_{2}\right)\right)<\mu_{5}$, then all the eigenvalues of the Jacobian matrix $J\left(E_{2}\right)$ near the equilibrium point $E_{2}$ have negative real parts. Thus, due to the Routh-Hurwitz criterion, the equilibrium point $E_{2}$ is locally asymptotically stable.

Proof. The Jacobian matrix of LDM model (1) at $E_{2}$ is

$$
J\left(E_{2}\right)=\left(\begin{array}{ccccc}
\frac{\beta_{1} L_{2} P_{2}}{\left(a+P_{2}\right)^{2}}-\frac{r P_{2}}{k}-\frac{\beta_{1} P_{2}}{a+P_{2}} & 0 & 0 & 0 \\
\frac{a e_{1} \beta_{1} L_{2}}{\left(a+P_{2}\right)^{2}} & 0 & 0 & 0 & -\frac{\beta_{2} L_{2}}{b+L_{2}} \\
0 & \varepsilon \alpha & -\mu_{3} & 0 & 0 \\
0 & (1-\varepsilon) \alpha \frac{\gamma \eta M_{2}}{\left(\eta+F_{2}\right)^{2}} & -\frac{\gamma \eta}{\eta+F_{2}}-\mu_{4} & 0 \\
0 & 0 & 0 & 0 & \frac{e_{2} \beta_{2} L_{2}}{b+L_{2}}-\mu_{5}
\end{array}\right) .
$$

The first three eigenvalues of $J\left(E_{2}\right)$ are $\lambda_{1}=-\mu_{3}$, $\lambda_{2}=-\left(\left((\gamma \eta) /\left(F_{2}+\eta\right)\right)+\mu_{4}\right), \quad$ and $\quad \lambda_{3}=\left(\left(e_{2} \beta_{2} L_{2}\right) /\right.$ $\left.\left(b+L_{2}\right)\right)-\mu_{5}$. The other roots are determined by

$$
\lambda^{2}+P_{2}\left(\frac{\beta_{1} L_{2}}{\left(a+P_{2}\right)^{2}}-\frac{r}{k}\right) \lambda+\frac{a e_{1} \beta_{1}^{2} P_{2} L_{2}}{\left(a+P_{2}\right)^{2}}=0 .
$$

When $\left(\left(\beta_{1} L_{2}\right) /\left(a+P_{2}\right)^{2}\right)<(r / k)$ and $\left(\left(e_{2} \beta_{2} L_{2}\right) /\right.$ $\left.\left(b+L_{2}\right)\right)<\mu_{5}$, then all the eigenvalues of the Jacobian matrix $J\left(E_{2}\right)$ near the equilibrium point $E_{2}$ have negative real parts. Thus, due to the Routh-Hurwitz criterion, the equilibrium point $E_{2}$ is locally asymptotically stable. 
The local stability of the coexistence equilibrium point $E_{3}=\left(P_{3}, L_{3}, F_{3}, M_{3}, W_{3}\right)$ is investigated as follows. The Jacobian matrix of model (1) at $E_{3}$ is

$$
J\left(E_{3}\right)=\left(\begin{array}{ccccc}
\frac{\beta_{1} L_{3} P_{3}}{\left(a+P_{3}\right)^{2}}-\frac{r P_{3}}{k} & -\frac{\beta_{1} P_{3}}{a+P_{3}} & 0 & 0 & 0 \\
\frac{a e_{1} \beta_{1} L_{3}}{\left(a+P_{3}\right)^{2}} & 0 & 0 & 0 & -\frac{L_{3} \beta_{2}}{b+L_{3}} \\
0 & \varepsilon \alpha_{1} & -\mu_{3} & 0 & 0 \\
0 & (1-\varepsilon) \alpha_{1} & \frac{\gamma \eta M_{3}}{\left(\eta+F_{3}\right)^{2}} & -\frac{\gamma \eta}{\eta+F_{3}}-\mu_{4} & 0 \\
0 & \frac{b e_{2} \beta_{2} W_{3}}{\left(b+L_{3}\right)^{2}} & 0 & 0 & 0
\end{array}\right) .
$$

The first two eigenvalues of $J\left(E_{2}\right)$ are $\lambda_{1}=-\mu_{3}$ and $\lambda_{2}=-\left((\gamma \eta) /\left(\eta+F_{2}\right)\right)-\mu_{4}$. The other eigenvalues are determined by

$$
\lambda^{3}+c_{1} \lambda^{2}+c_{2} \lambda+c_{3}=0
$$

where

$$
\begin{aligned}
& c_{1}=\frac{\beta_{1} L_{3} P_{3}}{\left(a+P_{3}\right)^{2}}-\frac{\beta_{2} L_{3} W_{3}}{\left(b+L_{3}\right)^{2}}-\frac{r P_{3}}{k}, \\
& c_{2}=L_{3}\left(\frac{a \beta_{1}^{2} e_{1} P_{3}}{\left(a+P_{3}\right)^{3}}+\frac{b \beta_{2}^{2} e_{2} W_{3}}{\left(b+L_{3}\right)^{3}}+\frac{\beta_{2} P_{3} r W_{3}}{k\left(b+L_{3}\right)^{2}}-\frac{\beta_{1} \beta_{2} L_{3} P_{3} W_{3}}{\left(a+P_{3}\right)^{2}\left(b+L_{3}\right)^{2}}\right), \\
& c_{3}=\frac{b \beta_{2}^{2} e_{2} L_{3} P_{3} W_{3}\left(\beta_{1} k L_{3}-r\left(a+P_{3}\right)^{2}\right)}{k\left(a+P_{3}\right)^{2}\left(b+L_{3}\right)^{3}},
\end{aligned}
$$

when $c_{1}>0, c_{3}>0$ and $c_{1} c_{2}>c_{3}$; then, all the eigenvalues of the Jacobian matrix $J_{3}$ near the coexistence equilibrium point $E_{3}$ have negative real parts. Hence, due to the Routh-Hurwitz criterion, the coexistence equilibrium point
$E_{3}$ is locally asymptotically stable. The local stability of the coexistence equilibrium point $E_{3}$ is investigated in the following theorem.

Theorem 7. If $c_{1}>0, c_{3}>0$ and $c_{1} c_{2}>c_{3}$, then the coexistence equilibrium point $E_{3}$ is locally asymptotically stable.

\section{Bifurcation Analysis}

In this section, the local bifurcations near the equilibrium points of lesser date moth model (1) are investigated with the help of Sotomayor's theorem [25] and the Hopf bifurcation theorem [26] to discuss the bifurcation analysis of the underlying system. LDM model (1) can be rewritten in a vector form $(\mathrm{d} X / \mathrm{d} t)=G(X)$, where $X=(P, L, F, M, W)^{T}$ and $G=$ $\left(f_{1}, f_{2}, f_{3}, f_{4}, f_{5}\right)^{T}$ with $f_{i}, i=1,2,3,4,5$ are given in the right hand side of LDM model (1). Following [27, 28], according to Jacobian matrix of LDM system, one can verify that for any nonzero vector $U=\left(\zeta_{1}, \zeta_{2}, \zeta_{3}, \zeta_{4}, \zeta_{5}\right)^{T}$ and $\varphi$ is any bifurcation parameter, and one obtains the following: 


$$
\begin{aligned}
D^{2} G(X, \varphi)(U, U)= & \frac{\partial^{2} G}{\partial P^{2}} \zeta_{1}^{2}+\frac{\partial^{2} G}{\partial L^{2}} \zeta_{2}^{2}+\frac{\partial^{2} G}{\partial f^{2}} \zeta_{3}^{2}+\frac{\partial^{2} G}{\partial M^{2}} \zeta_{4}^{2}+\frac{\partial^{2} G}{\partial W^{2}} \zeta_{5}^{2}+\frac{\partial^{2} G}{\partial P \partial L} \zeta_{1} \zeta_{2}+\frac{\partial^{2} G}{\partial L \partial P} \zeta_{2} \zeta_{1} \\
& +\frac{\partial^{2} G}{\partial P \partial F} \zeta_{1} \zeta_{3}+\frac{\partial^{2} G}{\partial F \partial P} \zeta_{3} \zeta_{1}+\frac{\partial^{2} G}{\partial P \partial M} \zeta_{1} \zeta_{4}+\frac{\partial^{2} G}{\partial M \partial P} \zeta_{4} \zeta_{1}+\frac{\partial^{2} G}{\partial P \partial W} \zeta_{1} \zeta_{5}+\frac{\partial^{2} G}{\partial W \partial P} \zeta_{5} \zeta_{1} \\
& +\frac{\partial^{2} G}{\partial L \partial F} \zeta_{2} \zeta_{3}+\frac{\partial^{2} G}{\partial F \partial L} \zeta_{3} \zeta_{2}+\frac{\partial^{2} G}{\partial L \partial M} \zeta_{2} \zeta_{4}+\frac{\partial^{2} G}{\partial M \partial L} \zeta_{4} \zeta_{2}+\frac{\partial^{2} G}{\partial L \partial W} \zeta_{2} \zeta_{5}+\frac{\partial^{2} G}{\partial W \partial L} \zeta_{5} \zeta_{2} \\
& +\frac{\partial^{2} G}{\partial F \partial M} \zeta_{3} \zeta_{4}+\frac{\partial^{2} G}{\partial M \partial f} \zeta_{4} \zeta_{3}+\frac{\partial^{2} G}{\partial F \partial W} \zeta_{3} \zeta_{5}+\frac{\partial^{2} G}{\partial W \partial F} \zeta_{5} \zeta_{3}+\frac{\partial^{2} G}{\partial M \partial W} \zeta_{4} \zeta_{5}+\frac{\partial^{2} G}{\partial W \partial M} \zeta_{5} \zeta_{4} .
\end{aligned}
$$

Consequently, we obtain that

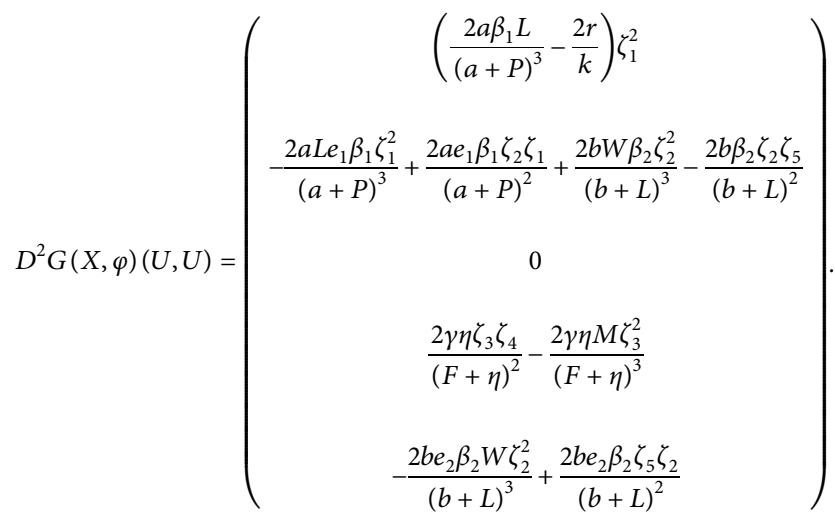

Theorem 8. LDM system (1) undergoes a transcritical bifurcation with respect to the bifurcation parameter $r$ around $E_{0}$ if $r=\mu_{1}$.

Proof. Let $V_{1}=\left(v_{1}, v_{2}, v_{3}, v_{4}, v_{5}\right)^{T}$ be the eigenvector corresponding to eigenvalue $\lambda=0$ of $J\left(E_{0}\right)$; hence, $J\left(E_{0}\right) V_{1}=0$ gives $V_{1}=\left(\nu_{1}, 0,0,0,0,\right)^{T}$, where $\nu_{1}$ is any nonzero real number. Similarly, suppose $V_{2}=\left(\tau_{1}, \tau_{2}, \tau_{3}, \tau_{4}, \tau_{5}\right)^{T}$ be the eigenvector corresponding to eigenvalue $\lambda=0$ of $J\left(E_{0}\right)^{T}$, thus $J\left(E_{0}\right)^{T} V_{2}=0$ gives $V_{2}=\left(\tau_{1}, 0,0,0,0\right)$, where $\tau_{1}$ is any nonzero real number. Consider $(\partial G / \partial r)=G_{r}(X, r)=$ $(P(1-(P / k)), 0,0,0,0)^{T}$, thus $V_{2}^{T} G_{r}\left(E_{0}, r^{*}\right)=0$. Therefore, according to Sotomayor's theorem for local bifurcation, LDM model (1) has no saddle-node bifurcation near $E_{0}$ at $r^{*}=\mu_{1}$.

Now,

$$
D G_{r}\left(E_{0}, r^{*}\right)=\left(\begin{array}{ccccc}
1 & 0 & 0 & 0 & 0 \\
0 & 0 & 0 & 0 & 0 \\
0 & 0 & 0 & 0 & 0 \\
0 & 0 & 0 & 0 & 0 \\
0 & 0 & 0 & 0 & 0
\end{array}\right),
$$

then $V_{2}^{T} D G_{r}\left(E_{0}, r^{*}\right) V_{1}=v_{1} \tau_{1} \neq 0$. Using (20), one obtains

$$
V_{2}^{T} D^{2} G(X, r)\left(V_{1}, V_{1}\right)=-\frac{\beta_{1} \zeta_{5} \zeta_{1}}{a}-\frac{2 r \zeta_{1}^{2}}{k} \neq 0 .
$$

Thus, according to Sotomayor's theorem, LDM system (1) has a transcritical bifurcation at $r^{*}=\mu_{1}$ as the parameter $r$ passes through the value $r^{*}$, thus the proof is complete.

Theorem 9. LDM system (1) undergoes a transcritical bifurcation with respect to the bifurcation parameter $\beta_{1}$ around $E_{1}=\left(P_{1}, 0,0,0,0\right)$ if $R_{0}=1$.

Proof. The Jacobian matrix of LDM system (1) at the free lesser date moth equilibrium point $E_{1}$ with $\beta_{1}=\beta_{1}^{*}=((a+$ $\left.\left.P_{1}\right)\left(\alpha+\mu_{2}\right) /\left(e_{1} P_{1}\right)\right)$ has zero eigenvalue taking the form

$$
J\left(E_{1}, \beta_{1}^{*}\right)=\left(\begin{array}{ccccc}
-\left(r-\mu_{1}\right) & \frac{-\beta_{1} P_{1}}{a+P_{1}} & 0 & 0 & 0 \\
0 & 0 & 0 & 0 & 0 \\
0 & \varepsilon \alpha & -\mu_{3} & 0 & 0 \\
0 & (1-\varepsilon) \alpha & 0 & -\gamma-\mu_{4} & 0 \\
0 & 0 & 0 & 0 & -\mu_{5}
\end{array}\right) .
$$

The eigenvector corresponding to $J\left(E_{1}\right) V_{3}=0$ is

$$
V_{3}=\left(\begin{array}{c}
-\frac{\left(\beta_{1} P_{1}\right) \nu_{2}}{\left(a+P_{1}\right)\left(r-\mu_{1}\right)} \\
v_{2} \\
\frac{(\varepsilon \alpha) \nu_{2}}{\mu_{3}} \\
\frac{((1-\varepsilon) \alpha) \nu_{2}}{\gamma+\mu_{4}} \\
0
\end{array}\right) \text {, }
$$

where $v_{2}$ is any nonzero real number. Similarly, the eigenvector corresponding to $J\left(E_{1}\right)^{T} V_{4}=0$ is given by $V_{4}=\left(0, \tau_{2}, 0,0,0\right)^{T}$, where $\tau_{2}$ is any nonzero real number. Consider $\quad\left(\partial G / \partial \beta_{1}\right)=G_{\beta_{1}}\left(X, \beta_{1}\right)=(((-P L) /(a+P))$, 
$\left.\left(\left(e_{1} P L\right) /(a+P)\right), 0,0,0\right)^{T}$, thus $V_{4}^{T} G_{\beta_{1}}\left(E_{1}, \beta_{1}^{*}\right)=0$. Therefore, according to Sotomayor's theorem for local bifurcation, LDM model (1) has no saddle-node bifurcation near $E_{1}$ at $\beta_{1}^{*}=\left(\left(\left(a+P_{1}\right)\left(\alpha+\mu_{2}\right)\right) /\left(e_{1} P_{1}\right)\right)$. Now,

$$
D G_{\beta_{1}}\left(E_{1}, \beta_{1}^{*}\right)=\left(\begin{array}{ccccc}
0 \frac{-\beta_{1} P_{1}}{a+P_{1}} & 0 & 0 & 0 \\
0 & \frac{e_{1} \beta_{1} P_{1}}{a+P_{1}} & 0 & 0 & 0 \\
0 & 0 & 0 & 0 & 0 \\
0 & 0 & 0 & 0 & 0 \\
0 & 0 & 0 & 0 & 0
\end{array}\right) \text {, }
$$

then $V_{4}^{T} D G_{\beta_{1}}\left(E_{1}, \beta_{1}^{*}\right) V_{3}=\left(\left(e_{1} P_{1} \nu_{1} \tau_{1}\right) /\left(a+P_{1}\right)\right) \neq 0$. Using (20), one obtains

$$
V_{4}^{T} D^{2} G\left(X, \beta_{1}\right)\left(V_{3}, V_{3}\right)=\frac{2 a \beta_{1} e_{1}}{\left(a+P_{1}\right)^{2}} \nu_{1} \nu_{2}-\frac{2 \beta_{2}}{b} \nu_{2} \nu_{5} \neq 0
$$

Thus, according to Sotomayor's theorem, LDM system (1) has a transcritical bifurcation at $\beta_{1}^{*}=\left(\left(\left(a+P_{1}\right)\right.\right.$ $\left.\left.\left(\alpha+\mu_{2}\right)\right) /\left(e_{1} P_{1}\right)\right)$, as the parameter $\beta_{1}$ passes through the value $\beta_{1}^{*}$, thus the proof is complete. It is interesting to note that $R_{0}=1$ is equivalent to $r^{*}=\left(\left(k \mu_{1}\left(\alpha-\beta_{1}\right.\right.\right.$ $\left.\left.\left.e_{1}+\mu_{2}\right)\right) /\left((a+k)\left(\alpha+\mu_{2}\right)-\beta_{1} e_{1} k\right)\right)$.

Theorem 10. LDM system (1) undergoes a transcritical bifurcation with respect to the bifurcation parameter $\beta_{2}$ around $E_{2}=\left(P_{1}, L_{1}, F_{1}, M_{1}, 0\right)$ if $\beta_{2}=\left(\left(\mu_{5}\left(b+L_{2}\right)\right) /\left(e_{2} L_{2}\right)\right)$.

Proof. The Jacobian matrix of LDM system (1) at the free lesser date moth equilibrium point $E_{2}$ with $\beta_{2}=$ $\beta_{2}^{*}=\left(\left(\mu_{5}\left(b+L_{2}\right)\right) /\left(e_{2} L_{2}\right)\right)$ has zero eigenvalue taking the form

$J\left(E_{2}, \beta_{2}^{*}\right)=\left(\begin{array}{ccccc}\frac{\beta_{1} P_{2} L_{2}}{\left(a+P_{2}\right)^{2}}-\frac{r P_{2}}{k}-\frac{\beta_{1} P_{2}}{a+P_{2}} & 0 & 0 & 0 \\ \frac{e_{1} a \beta_{1} L_{2}}{\left(a+P_{2}\right)^{2}} & 0 & 0 & 0 & -\frac{L_{2} \beta_{2}}{b+L_{2}} \\ 0 & \varepsilon \alpha & -\mu_{3} & 0 & 0 \\ 0 & (1-\varepsilon) \alpha \frac{\gamma \eta M_{2}}{\left(\eta+F_{2}\right)^{2}}-\frac{\gamma \eta}{\eta+F_{2}}-\mu_{4} & 0 \\ 0 & 0 & 0 & 0 & 0\end{array}\right)$

The eigenvector corresponding to $J\left(E_{1}\right) V_{5}=0$ is

$$
V_{5}=\left(\begin{array}{c}
v_{1} \\
\left(\frac{L_{2}}{a+P_{2}}-\frac{r\left(a+P_{2}\right)}{k \beta_{1}}\right) \nu_{1} \\
\frac{\left(\varepsilon \alpha_{1}\left(k L_{2} \beta_{1}-r\left(a+P_{2}\right)^{2}\right)\right) \nu_{1}}{k \beta_{1} \mu_{3}\left(a+P_{2}\right)} \\
\frac{\left(\alpha_{1}\left(k L_{2} \beta_{1}-r\left(a+P_{2}\right)^{2}\right)\left((1-\varepsilon) \mu_{3}\left(\eta+F_{2}\right)^{2}+\gamma \varepsilon \eta M_{2}\right)\right) \nu_{1}}{k\left(\eta+F_{2}\right)\left(a+P_{2}\right) \beta_{1} \mu_{3}\left(\gamma \eta+\left(\eta+F_{2}\right) \mu_{4}\right)} \\
\frac{\left(a e_{1} \beta_{1}\left(b+L_{2}\right)\right) \nu_{1}}{\beta_{2}\left(a+P_{2}\right)^{2}}
\end{array}\right),
$$

where $v_{1}$ is any nonzero real number. Similarly, the eigenvector corresponding to $J\left(E_{2}\right)^{T} V_{6}=0$ is given by $V_{6}=\left(0,0,0,0, \tau_{5}\right)^{T}$, where $\tau_{5}$ is any nonzero real number. Consider $\left(\partial G / \partial \beta_{2}\right)=G_{\beta_{2}}\left(X, \beta_{2}\right)=((0,((-L W) /(b+L))$, $\left.\left.0,0,0,\left(\left(e_{2} L W\right) /(b+L)\right)\right)\right)^{T}$, thus $V_{6}^{T} G_{\beta_{2}}\left(E_{2}, \beta_{2}^{*}\right)=0$. Therefore, according to Sotomayor's theorem for local bifurcation, LDM model (1) has no saddle-node bifurcation near $E_{2}$ at $\beta_{2}^{*}=\left(\left(\mu_{5}\left(b+L_{2}\right)\right) /\left(e_{2} L_{2}\right)\right)$. Now,

$$
D G_{\beta_{2}}\left(E_{2}, \beta_{2}^{*}\right)=\left(\begin{array}{ccccc}
0 & 0 & 0 & 0 & 0 \\
0 & 0 & 0 & 0 & \frac{-L_{2}}{b+L_{2}} \\
0 & 0 & 0 & 0 & 0 \\
0 & 0 & 0 & 0 & 0 \\
0 & 0 & 0 & 0 & \frac{e_{2} L_{2}}{b+L_{2}}
\end{array}\right) \text {, }
$$

then $V_{6}^{T} D G_{\beta_{2}}\left(E_{2}, \beta_{2}^{*}\right) V_{5}=\left(\left(a \beta_{1} e_{1} e_{2} L_{2} \nu_{1} \tau_{5}\right) / \beta_{2}\left(a+P_{2}\right)^{2}\right) \neq$ 0 . Using (20), one obtains

$$
V_{6}^{T} D^{2} G\left(X, \beta_{2}\right)\left(V_{5}, V_{5}\right)=\frac{2 b \beta_{2} e_{2} \nu_{2} \nu_{5} \tau_{5}}{\left(b+L_{2}\right)^{2}} \neq 0 .
$$

Thus, according to Sotomayor's theorem, LDM system (1) has a transcritical bifurcation at $\beta_{2}^{*}=\left(\left(\left(a+P_{1}\right)(\alpha+\right.\right.$ $\left.\left.\left.\mu_{2}\right)\right) /\left(e_{1} P_{1}\right)\right)$ as the parameter $\beta_{2}$ passes through the value $\beta_{2}^{*}=\left(\left(\mu_{5}\left(b+L_{2}\right)\right) /\left(e_{2} L_{2}\right)\right)$, thus the proof is complete.

In this part, we shall show that as the coexistence equilibrium $E_{3}$ loses stability, periodic solutions can bifurcate from the positive equilibrium. We choose the parameter $r$ as the bifurcation parameter as it plays a crucial role in the growth of date palm tree. We shall apply Liu's criteria [26] to obtain the conditions for periodic solution arising from Hopf bifurcation.

Theorem 11. For the coexistence equilibrium point $E_{3}$ of lesser date moth (1), the system around $E_{3}$ enters into the Hopf 
bifurcation when $r$ passes $r^{*}$ if the coefficients $c_{j}(r)(j=$ $1,2,3)$ at $r=r^{*}$ satisfy the following condition:

(1) $\Phi\left(r^{*}\right)=\left[c_{1}\left(r^{*}\right) c_{2}\left(r^{*}\right)-c_{3}\left(r^{*}\right)\right]=0$

(2) $(d \Phi(r) / d \varphi) \mid r=r^{*} \neq 0$

The characteristic equation (15) of the coexistence equilibrium point $E_{3}$ has two purely imaginary roots if and only if $c_{1} c_{2}-c_{3}=0$. The value of $\Phi(r)=c_{1} c_{2}-c_{3}$ is given by

$$
\begin{aligned}
\Phi(r)= & L_{3}\left(( B _ { 1 1 } - \frac { \beta _ { 2 } L _ { 3 } W _ { 3 } } { ( b + L _ { 3 } ) ^ { 2 } } ) \left(\frac{a \beta_{1}^{2} e_{1} P_{3}}{\left(a+P_{3}\right)^{3}}\right.\right. \\
& \left.\left.+\frac{\beta_{2} W_{3}\left(b \beta_{2} e_{2}-B_{11}\left(b+L_{3}\right)\right)}{\left(b+L_{3}\right)^{3}}\right)-\frac{b \beta_{2}^{2} B_{11} e_{2} W_{3}}{\left(b+L_{3}\right)^{3}}\right),
\end{aligned}
$$

where $B_{11}=P_{3}\left((r / k)-\left(\left(\beta_{1} L_{3} P_{3}\right) /\left(a+P_{3}\right)^{2}\right)\right)$. For $r=r^{*}$, the characteristic equation (15) turns into

$$
\left(\lambda+c_{1}\right)\left(\lambda^{2}+c_{2}\right)=0
$$

The roots of equation (32) are $\lambda_{1}=i c_{2}, \lambda_{2}=-i c_{2}$, and $\lambda_{3}=c_{1}$. One can rewrite the roots as follows: $\lambda_{1}=m(r)+i n(r), \lambda_{2}=m(r)-i n(r)$, and $\lambda_{3}=-c_{1}$. To apply Liu's Hopf bifurcation criterion [26], we need to verify the transversality condition. Substituting $\lambda=m(r)+i n(r)$ into (15) and separating the real and imaginary, we get

$$
\begin{gathered}
m^{3}-3 m n^{2}+c_{1} m^{2}-c_{1} n^{2}+c_{2} m+c_{3}=0, \\
n^{2}=3 m^{2}+2 c_{1} m+c_{2} .
\end{gathered}
$$

Substituting the value of $n^{2}$ from (34) into (33), differentiating with respect to $r$, and utilizing $m\left(r^{*}\right)=0$ and $n\left(r^{*}\right) \neq 0$, we have

$$
\frac{\mathrm{d} m}{\mathrm{~d} r} \mid r=r^{*}=\left[\frac{(\mathrm{d} / \mathrm{d} r)\left(c_{1} c_{2}-c_{3}\right)}{2\left(c_{1}^{2}+c_{2}\right)}\right]_{r=r^{*}}=\left(\frac{(\mathrm{d} \Phi(r) / \mathrm{d} r)}{2\left(c_{1}^{2}+c_{2}\right)}\right)_{r=r^{*}} \neq 0 .
$$

Thus, it can be concluded that the coexistence equilibrium point $E_{3}$ is locally asymptotically stable for $r<r^{*}$. Furthermore, according to Liu's criterion, a simple Hopf bifurcation occurs at $r=r^{*}$, and for $r>r^{*}$, the interior equilibrium point $E_{3}$ approaches to a periodic solution. According to Theorem 11, there exists a Hopf bifurcation in lesser date moth model (1) where the Hopf bifurcation is controlled by $r$.

\section{Numerical Simulations}

In this part, numerical simulations of lesser date moth system (1) are conducted to illustrate the theoretical results obtained before. The interactions between date palm, lesser date moth, and natural enemy will be simulated by the following parameters:

$$
\begin{aligned}
r & =3, \\
k & =3, \\
\mu_{1} & =0.01, \\
\mu_{2} & =0.03, \\
\mu_{3} & =0.01, \\
\mu_{4} & =0.01, \\
\mu_{5} & =0.01, \\
\alpha & =0.5, \\
\beta_{1} & =2, \\
\beta_{2} & =0.5, \\
\gamma & =0.5, \\
\eta & =0.2, \\
\varepsilon & =0.5, \\
a & =3, \\
b & =10 .
\end{aligned}
$$

From Figure 1, it can be seen that the sex pheromone trap parameter $\eta$ is important in that it affects the population density of the lesser date moth male. One can observe from Figure 1 that the population density of lesser date moth male decreases with increasing $\eta$. We conclude that the dynamics of lesser date moth can be controlled by sex pheromone trap parameters $\eta$. In order to show the effect of the intrinsic growth rate of the date palm tree $r$, we draw the bifurcation diagram with respect to $r$ as a bifurcation parameter. If we increase the value of growth rate $r$ and keeping all other parameter value fixes, it can be seen that a transcritical bifurcation occurs at $r=0.01$, as shown in Figure 2 and stated in Theorem 8. It can also be observed that when $0.01<r<0.0198148$, the lesser date moth free equilibrium point $E 1=\left(P_{1}, 0,0,0,0\right)$ is locally asymptotically stable. It can be seen that a transcritical bifurcation occurs at $r=0.0198148$, as shown in Figure 2 and stated in Theorem 9. To understand the change in the dynamical behavior of the lesser date moth system model system (1) due to variation of the predation intensity between date palm and larvae $\beta_{1}$, we increase the value of $\beta_{1}$ and keep all other parameter value fixes. It can be seen that a transcritical bifurcation occurs at $\beta_{1}=1.32722$, as shown in Figure 3 and stated in Theorem 9. In order to show the effect of predation intensity between larvae and natural enemy $\beta_{2}$, we draw the bifurcation diagram with respect to $\beta_{2}$ as a bifurcation parameter. If we increase the value of predation rate $\beta_{2}$ and keeping all other parameter value fixes, it can be seen that a transcritical bifurcation occurs at $\beta_{2}=0.132143$, as shown in Figure 4 and stated in Theorem 10. The bifurcation diagram with respect to $r$ as a bifurcation parameter is indicated in Figure 5. According to Theorem 11, it can be seen that the supercritical Hopf bifurcation value for $F$ and $W$ is localized at $r=6.13149$ and one can verify the results $c_{1}(6.13149)=$ $5.74251>0, c_{3}(6.13149)=0.0136073>0$, and $\Phi(6.13149)$ 


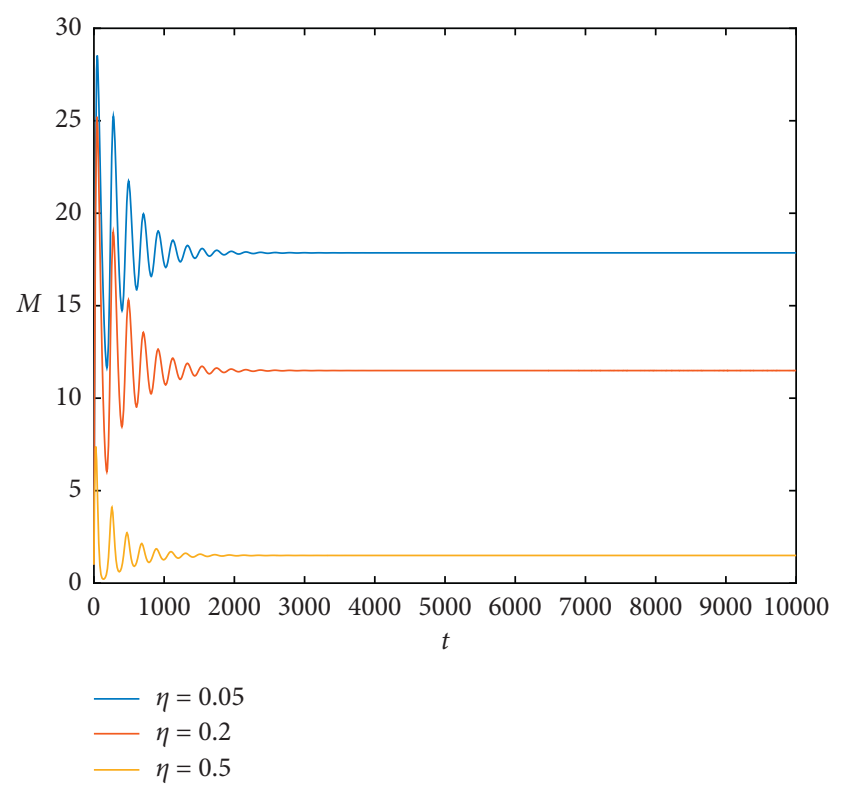

Figure 1: Time series of model (1) with. $\eta=0.05,0.2,0.5$.

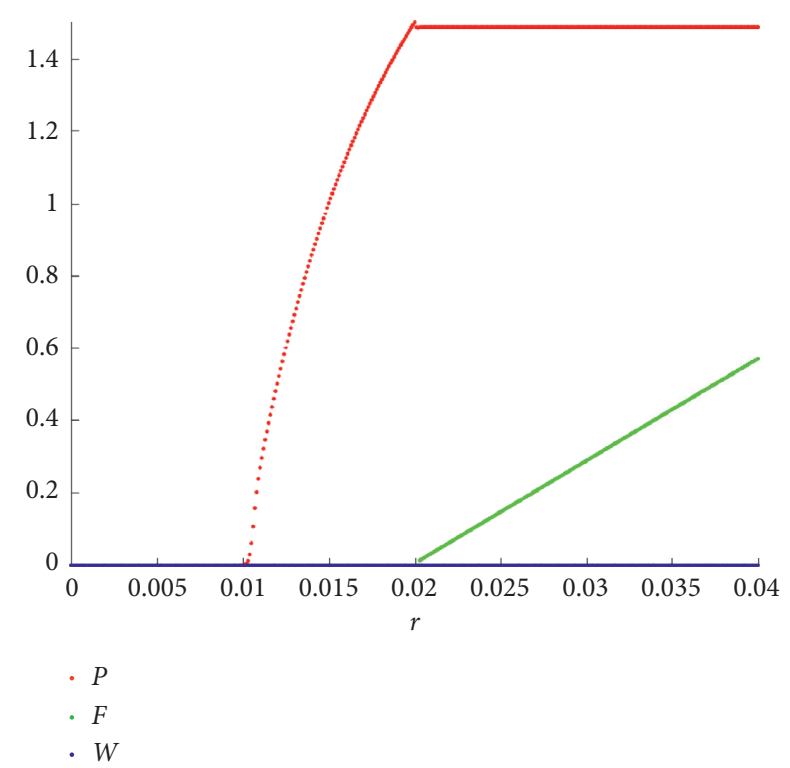

FIGURE 2: Bifurcation diagram of lesser date moth model (1) with respect to $r, 0 \leq r \leq 0.04$

$=0$. Furthermore, $(\mathrm{d} \Phi(r) / \mathrm{d} r)=-0.105178$, thus the transversality condition for Hopf bifurcation is also satisfied. Figure 6 shows stable steady distribution for $r=0.5$. Now, if we increase the values of $r$ from 5 to 7 , we observe that the system becomes unstable, and the lesser date moth model shows limit cycle oscillations. It is clear that if we increase the increase date palm tree intrinsic growth rate, the system enters into Hopf bifurcation. In order to show the effect of carrying capacity around the coexistence

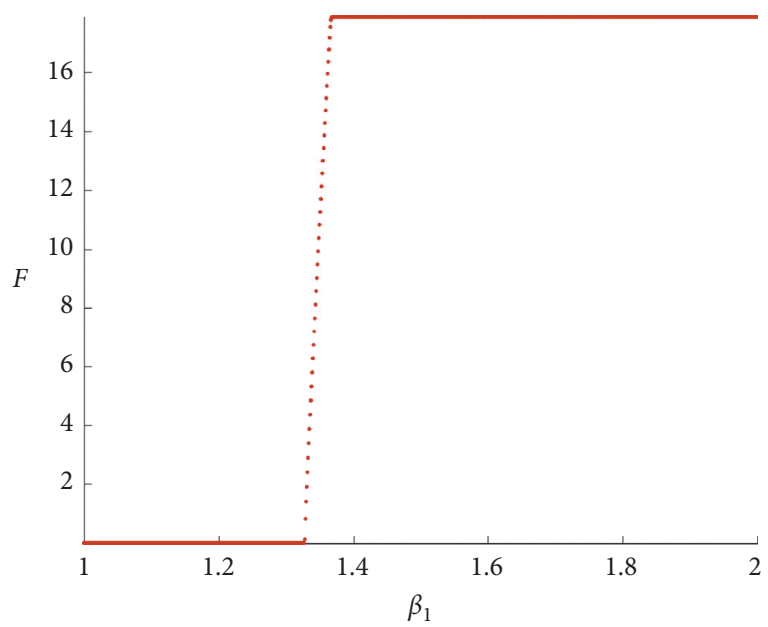

Figure 3: Bifurcation diagram of lesser date moth model (1) with respect to $\beta_{1}$.

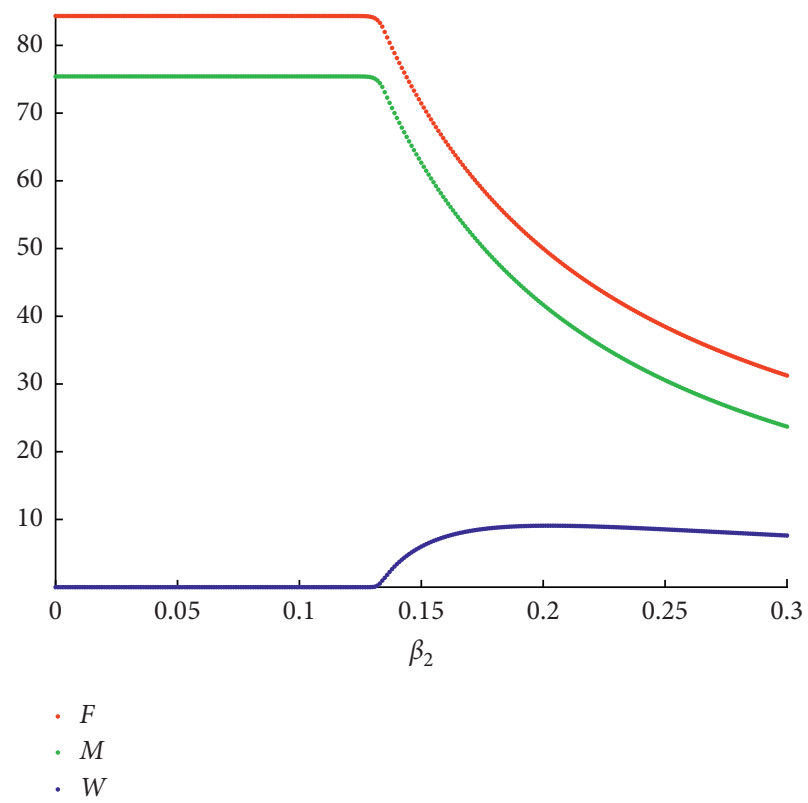

Figure 4: Bifurcation diagram of lesser date moth model (1) with respect to $\beta_{2}$.

equilibrium points, we draw the bifurcation diagram with respect to $k$ as a bifurcation parameter. It can be seen that the supercritical Hopf bifurcation value for $F$ localized at $k=4.68754$, as shown in Figure 7. It can also be observed that when $k<4.68754$, the coexistence equilibrium point $E_{3}$ is locally asymptotically stable, as shown in Figure 8(a). For $k>4.68754$, the system undergoes limit cycle behavior, as shown in Figure 8(b). 


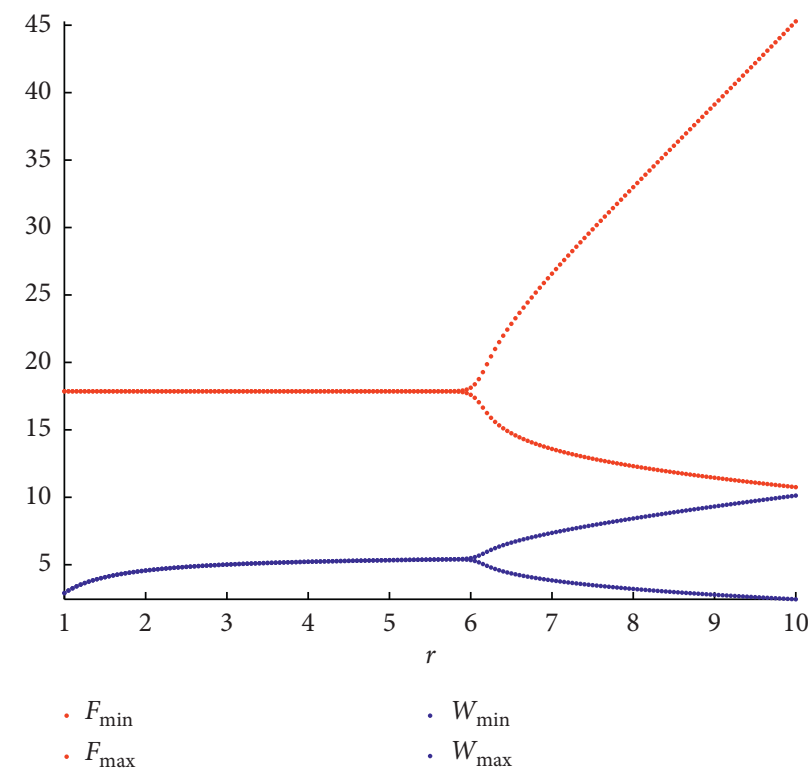

Figure 5: Bifurcation diagram of lesser date moth model (1) with respect to $r, 1 \leq r \leq 10$.
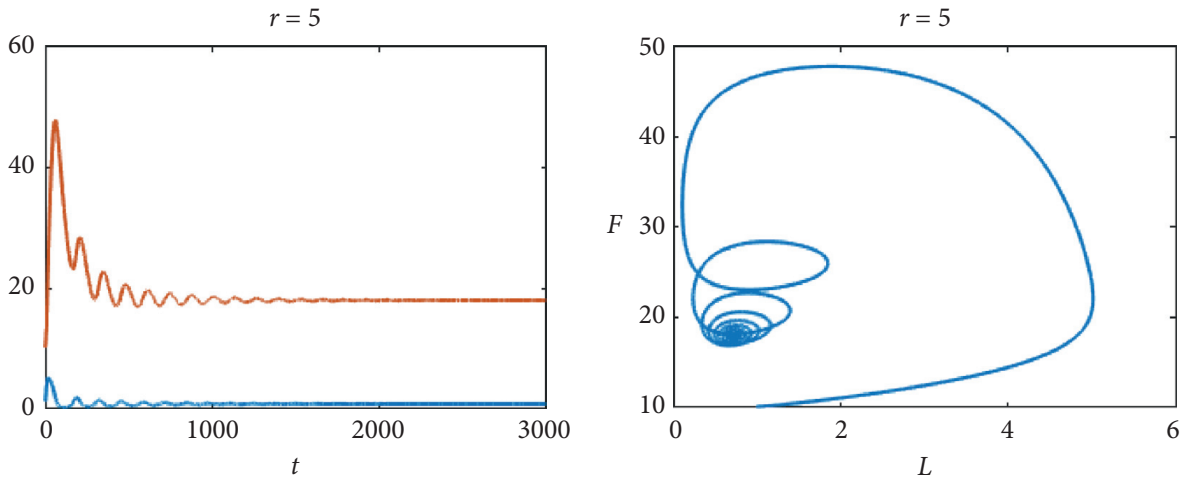

$L$
$-F$

(a)
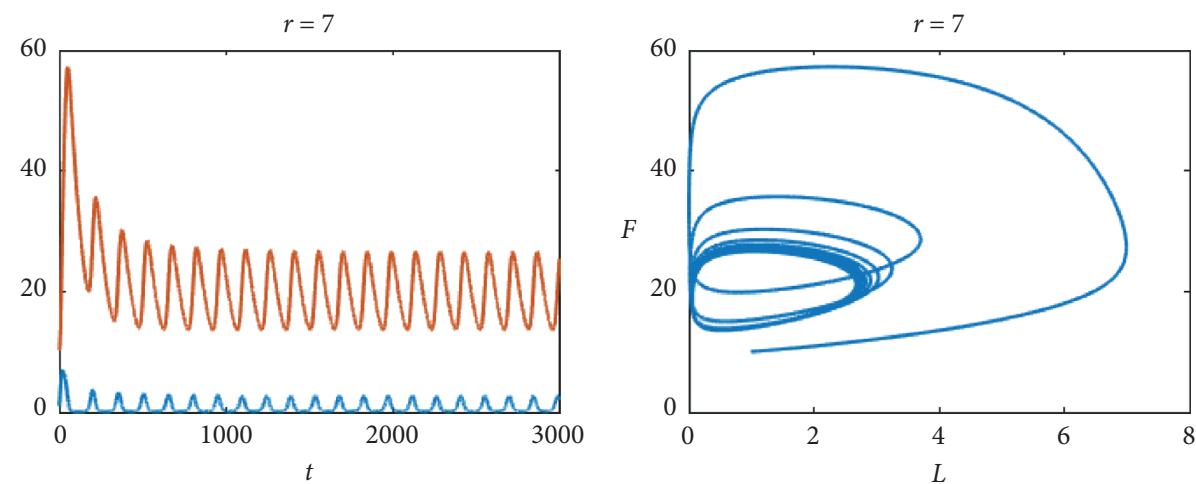

$-L$

(b)

FIgURE 6: Time series of model (1) with $r=5,7$. 


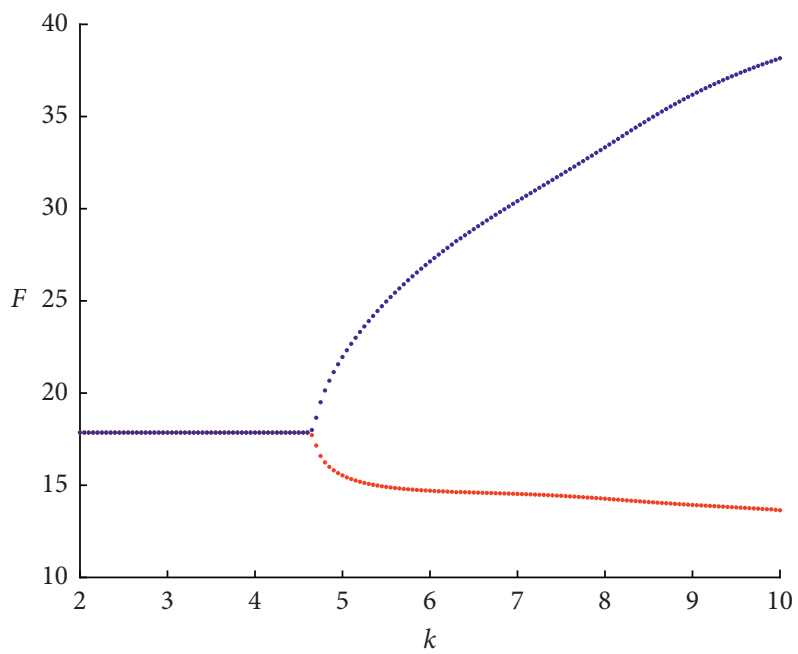

Figure 7: Bifurcation diagram of lesser date moth model (1) with respect to $k$.

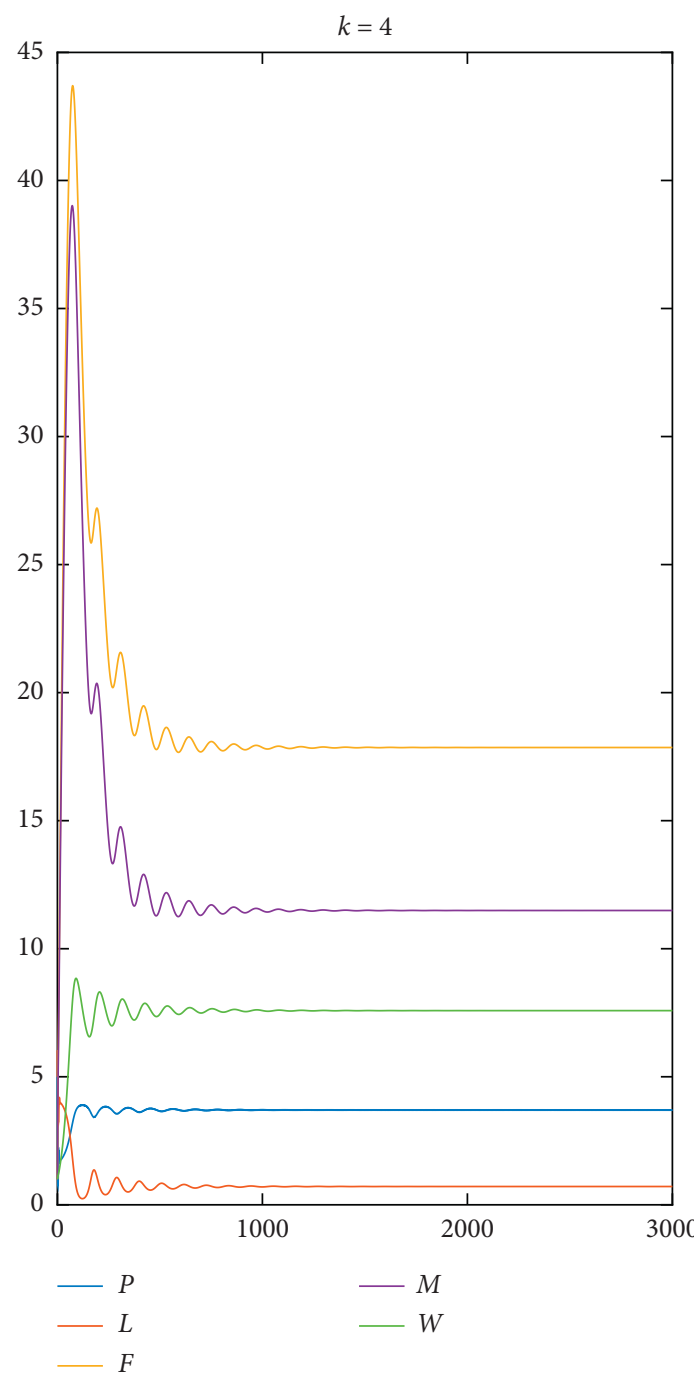

(a)

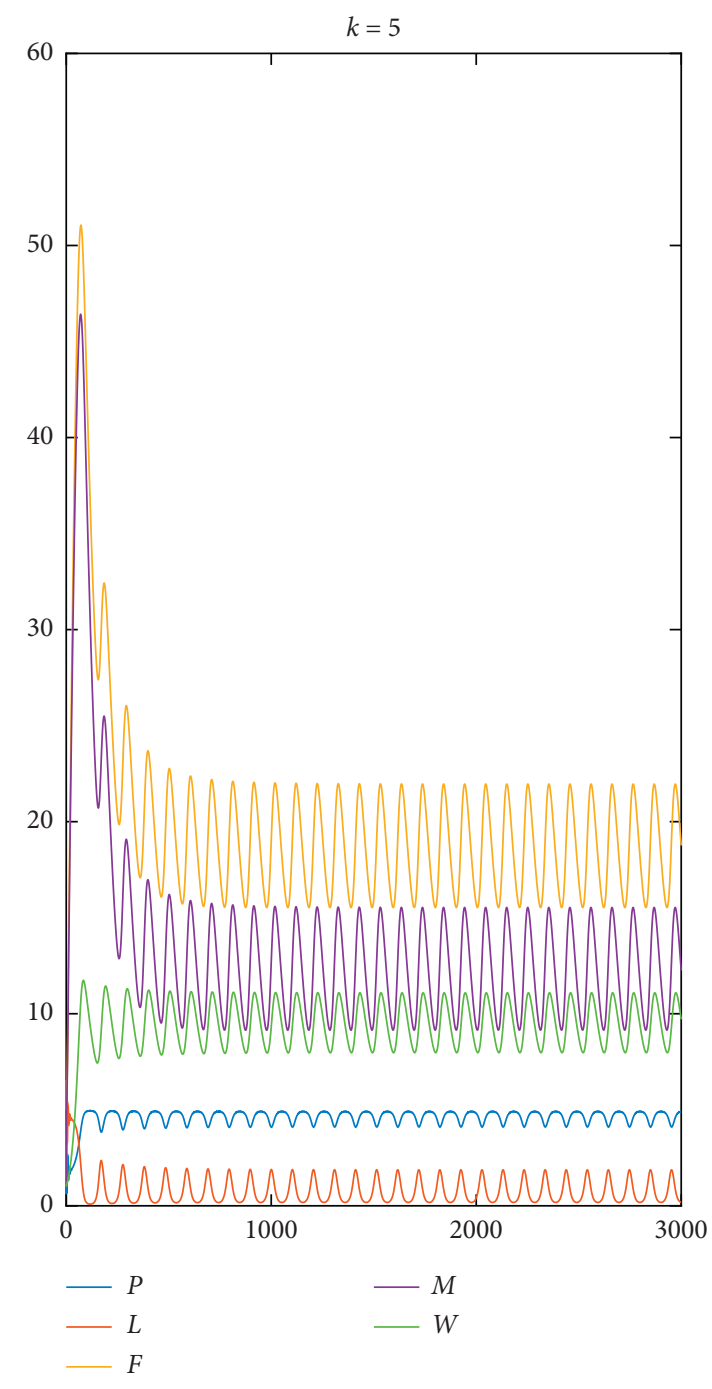

(b)

Figure 8: Time series of model (1) with $k=4,5$. (a) $k=4$. (b) $k=5$. 


\section{Discussion and Conclusion}

In this paper, we consider a mathematical model for lesser date moth using sex pheromone traps. We have investigated the sex pheromone trap impact on the spread of lesser date moth in date palm trees. The interaction between date palm tree, lesser date moth, and the natural enemy was investigated. We obtain some sufficient conditions that ensure the local and global stability of equilibrium points. It has been shown that for $R_{0}<1$, the equilibrium $E_{1}$ is locally asymptotically stable. According to Theorem 9, we may conclude that for $R_{0}=1$, the LDM model undergoes a transcritical bifurcation. According to Theorem 10, there exists a Hopf bifurcation in the lesser date moth model where the Hopf bifurcation is controlled by the date palm tree intrinsic growth rate $r$. We conclude that sex pheromone trap parameters can control the dynamics of lesser date moth. The occurrence of local bifurcation near the equilibrium point is performed using Sotomayor's theorem. Analytical studies can never be completed without numerical illustrations of the analytical results. So, all analytical findings are numerically verified. The numerical studies in this paper show the system's dynamical behavior at equilibrium points, which is in good agreement with the analytical studies. We have noticed that the sex pheromone trap parameter $\eta$ plays a key role in controlling the population density of the lesser date moth male. It is worth noting that although we presented a realistic mathematical model on the spread of the lesser date moth and its control using pheromone traps and natural enemy, our study is only limited to a comprehensive theoretical analysis of such scenarios. Our theoretical study can capture the main mechanisms associated with lesser date moth control using pheromone traps with no chemical pesticides or mechanical resistance to the insect. A mathematical model representing the integrated lesser date moth control using pheromone traps, insecticides, and mechanical resistance would benefit significantly. As part of future work to improve the model of this paper, the model studied here could be refined to describe and explain the role of pesticides in LDM control in the presence of pheromone traps. Additional experimental or field investigations in this direction may be useful to verify whether or not the processes defined by the model are actually operating similarly in the real world. We assumed the environmental effect and temperature, and all other situations are homogeneous in the present work, but it is inhomogeneous in reality. One may consider a heterogeneous lesser date moth system for future work.

\section{Data Availability}

Data sharing is not applicable to this article as no datasets were generated or analyzed during the current study.

\section{Conflicts of Interest}

The authors declare that there are no conflicts of interest.

\section{Authors' Contributions}

All authors contributed equally to the writing of this paper. All authors read and approved the final manuscript.

\section{Acknowledgments}

The authors gratefully acknowledge Qassim University, represented by the Deanship of Scientific Research, for the financial support under the project number (cosao-bs2019-2-2-I-5469) during the academic year $1440 \mathrm{AH} / 2019$ AD.

\section{References}

[1] L. I. El-Juhany et al., "Degradation of date palm trees and date production in arab countries: causes and potential rehabilitation," Australian Journal of Basic and Applied Sciences, vol. 4, no. 8, pp. 3998-4010, 2010.

[2] J. Al-Khayri, "Date palm phoenix dactylifera. micropropagation," in Protocols for Micropropagation of Woody Trees and Fruits, pp. 509-526, Springer, 2007.

[3] A. Levi-Zada, D. Fefer, L. Anshelevitch et al., "Identification of the sex pheromone of the lesser date moth, batrachedra amydraula, using sequential spme auto-sampling," Tetrahedron Letters, vol. 52, no. 35, pp. 4550-4553, 2011.

[4] M. A. Al-Deeb and H. A. Al-Dhaheri, Use of a PheromoneBaited Trap to Monitor the Population of the Lesser Date Moth Batrachedra Amydraula (Lepidoptera: Batrachedridae) in the $U A E$, vol. 6, pp. 2572-2575, 2017.

[5] M. S. Hoddle, A. H. Al-Abbad, H. A. F. El-Shafie, J. R. Faleiro, A. A. Sallam, and C. D. Hoddle, "Assessing the impact of areawide pheromone trapping, pesticide applications, and eradication of infested date palms for rhynchophorus ferrugineus (coleoptera: Curculionidae) management in al ghowaybah, Saudi Arabia," Crop Protection, vol. 53, pp. 152-160, 2013.

[6] R. Carde, Using Pheromones to Disrupt Mating of Moth Pests, Perspectives in Ecological Theory and Integrated Pest Management, pp. 122-169, Cambridge University Press, Cambridge, UK, 2007.

[7] W. Wakil, J. R. Faleiro, and T. A. Miller, Sustainable Pest Management in Date Palm: Current Status and Emerging Challenges, Springer, Berlin, Germany, 2015.

[8] P. Witzgall, P. Kirsch, and A. Cork, "Sex pheromones and their impact on pest management," Journal of Chemical Ecology, vol. 36, no. 1, pp. 80-100, 2010.

[9] H. J. Barclay and G. J. R. Judd, "Models for mating disruption by means of pheromone for insect pest control," Researches on Population Ecology, vol. 37, no. 2, pp. 239-247, 1995.

[10] E. Sadeghi, V. Baniameri, A. Marouf et al., "Oviposition behaviour of goniozus swirskiana (hymenoptera: bethylidae: Bethylinae) a parasitoid of batrachedra amydraula meyrick from the warmest desert of Iran," World Applied Sciences Journal, vol. 20, no. 11, pp. 1493-1498, 2012.

[11] M. Abbas, S. Al-Khatry, R. Shidi, and N. A. Al-Ajmi, "Natural enemies of the lesser date moth, batrachedra amydraula meyrick (lepidoptera: batrachedridae) with special reference to its parasitoid goniozus sp," Egyptian Journal of Biological Pest Control, vol. 24, no. 2, p. 293, 2014.

[12] H. Barclay and P. Van den Driessche, "Pheromone trapping models for insect pest control," Researches on Population Ecology, vol. 25, no. 1, pp. 105-115, 1983.

[13] H. J. Barclay and J. Hendrichs, "Models for assessing the male annihilation of bactrocera spp. with methyl eugenol baits," Annals of the Entomological Society of America, vol. 107, no. 1, pp. 81-96, 2014.

[14] R. Anguelov, C. Dufourd, and Y. Dumont, "Mathematical model for pest-insect control using mating disruption and 
trapping," Applied Mathematical Modelling, vol. 52, pp. 437-457, 2017.

[15] J. P. Ntahomvukiye, A. Temgoua, and S. Bowong, "Study of the population dynamics of busseola fusca, maize pest," Acta Biotheoretica, vol. 66, no. 4, pp. 379-397, 2018.

[16] S. Xiang, Y. Pei, and X. Liang, "Analysis and optimization based on a sex pheromone and pesticide pest model with gestation delay," International Journal of Biomathematics, vol. 12, no. 5, Article ID 1950054, 2019.

[17] M. D. Tapi, L. Bagny-Beilhe, and Y. Dumont, "Miridae control using sex-pheromone traps. modeling, analysis and simulations," Nonlinear Analysis: Real World Applications, vol. 54, Article ID 103082, 2020.

[18] A. Das and G. P. Samanta, "A prey-predator model with refuge for prey and additional food for predator in a fluctuating environment," Physica A: Statistical Mechanics and Its Applications, vol. 538, Article ID 122844, 2020.

[19] S. G. Mortoja, P. Panja, A. Paul, S. Bhattacharya, and S. K. Mondal, "Is the intermediate predator a key regulator of a tri-trophic food chain model?: An illustration through a new functional response," Chaos, Solitons and Fractals, vol. 132, Article ID 109613, 2020.

[20] A. A. Elsadany, A. E. Matouk, A. G. Abdelwahab, and H. S. Abdallah, "Dynamical analysis, linear feedback control and synchronization of a generalized lotka-volterra system," International Journal of Dynamics and Control, vol. 6, no. 1, pp. 328-338, 2018.

[21] Y. Louartassi, A. Alla, K. Hattaf, and A. Nabil, "Dynamics of a predator-prey model with harvesting and reserve area for prey in the presence of competition and toxicity," Journal of Applied Mathematics and Computing, vol. 59, no. 1-2, pp. 305-321, 2019.

[22] P. Van den Driessche and J. Watmough, "Reproduction numbers and sub-threshold endemic equilibria for compartmental models of disease transmission," Mathematical Biosciences, vol. 180, no. 1-2, pp. 29-48, 2002.

[23] A. J. Alqarni, A. S. Rambely, and I. Hashim, "Dynamic modelling of interactions between microglia and endogenous neural stem cells in the brain during a stroke," Mathematics, vol. 8, no. 1, p. 132, 2020.

[24] K. Manna, V. Volpert, and M. Banerjee, "Dynamics of a diffusive two-prey-one-predator model with nonlocal intraspecific competition for both the prey species," Mathematics, vol. 8 , no. 1 , p. 101, 2020.

[25] L. Perko, Differential Equations And Dynamical Systems, Vol. 7, Springer Science and Business Media, Berlin, Germany, 2013.

[26] W. M. Liu, "Criterion of hopf bifurcations without using eigenvalues," Journal of Mathematical Analysis and Applications, vol. 182, no. 1, pp. 250-256, 1994.

[27] C. Maji, D. Mukherjee, and D. Kesh, "Deterministic and stochastic analysis of an eco-epidemiological model," Journal of Biological Physics, vol. 44, no. 1, pp. 17-36, 2018.

[28] M. Haque, J. King, J. R. King, S. Preston, M. Loose, and D. de Pomerai, "Mathematical modelling of a micrornaregulated gene network in caenorhabditis elegans," Mathematical Biosciences and Engineering, vol. 17, no. 4, pp. 2881-2904, 2020. 